IZA DP No. 5158

Political Culture and Discrimination in Contests

Gil S. Epstein

Yosef Mealem

Shmuel Nitzan

August 2010 


\title{
Political Culture and Discrimination in Contests
}

\author{
Gil S. Epstein \\ Bar-llan University, \\ CReAM and IZA
}

\section{Yosef Mealem}

Netanya Academic College

\author{
Shmuel Nitzan \\ Bar-Ilan University
}

\author{
Discussion Paper No. 5158 \\ August 2010
}

\author{
IZA \\ P.O. Box 7240 \\ 53072 Bonn \\ Germany \\ Phone: +49-228-3894-0 \\ Fax: +49-228-3894-180 \\ E-mail: iza@iza.org
}

\begin{abstract}
Any opinions expressed here are those of the author(s) and not those of IZA. Research published in this series may include views on policy, but the institute itself takes no institutional policy positions.

The Institute for the Study of Labor (IZA) in Bonn is a local and virtual international research center and a place of communication between science, politics and business. IZA is an independent nonprofit organization supported by Deutsche Post Foundation. The center is associated with the University of Bonn and offers a stimulating research environment through its international network, workshops and conferences, data service, project support, research visits and doctoral program. IZA engages in (i) original and internationally competitive research in all fields of labor economics, (ii) development of policy concepts, and (iii) dissemination of research results and concepts to the interested public.
\end{abstract}

IZA Discussion Papers often represent preliminary work and are circulated to encourage discussion. Citation of such a paper should account for its provisional character. A revised version may be available directly from the author. 
IZA Discussion Paper No. 5158

August 2010

\section{ABSTRACT}

\section{Political Culture and Discrimination in Contests}

Many economic and political decisions are the outcome of strategic contests for a given prize. The nature of such contests can be determined by a designer who is driven by political considerations with a specific political culture. The main objective of this study is to analyze the effect of political culture and of valuation asymmetry on discrimination between the contestants. The weights assigned to the public well being and the contestants' efforts represent the political culture while discrimination is an endogenous variable that characterizes the mechanism allocating the prize. We consider situations under which the optimal bias of the designer is in favor of the contestant with the larger or smaller prize valuation and examine the effect of changes in the political culture and in valuation asymmetry on the designer's preferred discrimination between the contestants. Focusing on the two most widely studied types of contest success functions (deterministic all-pay-auctions and logit CSFs), we show that an all-pay auction is always the preferred CSF from the point of view of the contest designer. This result provides a new political-economic micro foundation to some of the most commonly used models in the contest literature.

JEL Classification: $\quad \mathrm{HO}$

Keywords: rent seeking political culture, discrimination, contests, logit contest success function, all-pay-auction

Corresponding author:

Gil S. Epstein

Department of Economics

Bar-llan University

52900 Ramat-Gan

Israel

E-mail: epsteig@mail.biu.ac.il

\footnotetext{
* Financial support from the Adar Foundation of the Economics Department of Bar-llan University is gratefully acknowledged.
} 


\section{Introduction}

Endogenous determination of contests may relate to all relevant elements of contests. This is obviously true with respect to the actions taken by the contestants. ${ }^{2}$ There are institutional elements, however, that are typically determined by contest designers; economic and political entrepreneurs. Such characteristics include the contest prize, the set of contestants, the structure of multi-stage contests, caps on political lobbying and the contest success function. ${ }^{3}$

Most of the literature on optimal contest design has focused on the choice of contest characteristics assuming that the designer's objective function depends on the contestants' efforts. Few attempts have been made to study the relationship between the designed contest and more general objective functions that take into account not only the efforts incurred by the contestants. ${ }^{4}$ Examining the endogenous determination of public policy that determines, in turn, the contestants' prizes or stakes, Epstein and Nitzan (2002), (2006a), (2006b), (2007), allow the designer's objective function to depend on the efforts of the contestants and on their expected aggregate utility. The weights assigned to these variables represent the political culture of the contest designers.

The current paper departs from the literature in three ways. First, it adds discrimination to the contest designer's tool box containing the possible means (control variables) to enhance his interest. Second, it applies the more general objective function proposed in Epstein and Nitzan (2002), (2006a), (2006b) to study the relationship between discrimination and political culture. Third, it compares the two most widely studied types of contest success functions (all-pay-auctions and logit

\footnotetext{
${ }^{2}$ Notably these actions include their efforts, Congleton, Hillman and Konrad (2008), Konrad (2009) and to their decisions to enter the contest, Morgan, Orzen and Sefton (2008). Other contest characteristics determined by the contestants are the contest coalition formation, Bloch et al. (2006), Sanchez-Pages (2007), and, within collective or group contests, the sharing rules of the prize among the group members, in case the prize is won, Baik (2008), Baik and Lee (2001), Nitzan and Ueda (2009), Ueda (2002).

${ }^{3}$ Glazer and Hassin (1988), Moldovanu and Sela (2001) and Nti (2004) deal with the contest prize, Baye et al. (1993), Amegashie (2000) and Taylor (1995) focus on the set of contestants, Gradstein (1998), Gradstein and Konrad (1999) and Amegashie (2000) examine the structure of multi-stage contests, Che and Gale (1998) study caps on lobbying and Nti (1997), (2004) and Kahana and Nitzan (2002) are concerned with the contest success function.

${ }^{4}$ Studying the endogenous determination of the optimal prize, Runkel (2006) and Singh and Wittman (1998) consider a designer's payoff function that depends on the performance of the contestants and on the difference in their winning probabilities (the closeness of the contest). This difference represents the uncertainty of the contest outcome that affects the interest it arouses and, in turn, the size of the contest audience. Dasgupta and Nti (1998) consider the endogenous determination of the contest success function, assuming that the designer's objective function depends on aggregate efforts and on his own valuation of the prize that may induce him not to award the prize.
} 
CSFs) under optimal discrimination. Such comparison has previously been carried out assuming either no discrimination, Che and Gale (1997), or some given, not necessarily optimal, discrimination, Epstein and Nitzan (2006b). Our main objective then is to analyze the effect of political culture and of asymmetry in the contestants' valuations of the prize on discrimination among the contestants and on the comparison between all-pay-auctions and logit CSFs.

Discrimination is often controlled by contest designers and our initial task is to clarify why this is indeed the case in the complex institutional environments where real contests are held. We also wish to justify our focus on the two most widely studied types of contest success functions. Our explanations of the empirical relevance of the particular design problem on which we focus are based on the economic and legal characteristics of the contest environment.

Many job openings and outsourcing of projects in the public sector need to be announced publicly in order to enable any interested potentially deserving candidate to take part in the contest on the position or project. By law, everyone is entitled to fair opportunity to take part and win the contest. However, each job or project has its specific required necessary conditions. These conditions are part of the contest requirements. The fact that the bureaucrat/regulator is responsible to determine and announce these requirements implies that he can control not only the group of contestants but, as in our abstract setting, give some candidate some known advantage over others. This is the basic reason why the bureaucrat is conceived as the contest designer. The designer can inform the contestants about the bias by stating, before the contest is held, the features that make a contestant advantageous (more likely to win). For example, the designer may state that he prefers the winner to have a strong financial background or sufficient administrative experience. Such open statement enables the designer to let the contestants know the bias in favor of one of them. However, this does not mean that the favored contestant certainly wins. It just means that the designer favors one contestant who is more likely to win if making the same effort as his rival. The bias is represented by the different values of a unit of effort made by the contestants. In the case of a conglomerate competing for a project, the designer may increase the winning chances of a different small group by adding specific requirements that give it an advantage over the conglomerate. He may declare, for instance, that firms with green technology experience have an advantage in the contest. In such a case, if the conglomerate does not have the required 
experience while the small firm does, the designer makes the contest less naturally biased in favor of the conglomerate (due to its size). By doing so, the designer can make the contest more equal (competitive) in order to increase the outlays of the contestants.

The legal right to conduct the contest and discriminate among the contestants by ensuring that the contest requirements are fulfilled affects the contest outcome and gives the contest designer a lot of power. This power can be used to promote the intended goal of the law (enhancement of the public welfare) or the designer's narrow objectives (maximization of the contestants' efforts, sewing a job for a specific candidate). The empirical relevance of the problem of possible abuse of the designer's power is clear. Recent evidence from Israel can serve as an illustration. The 2007 Israeli State Comptroller's Report refers to contests designed or tailor-made for specific candidates, as well as to conflicts of interest or biased tenders in many projects administered by the Haifa Port. More recently, in light of the potential misconduct of a designer who can manipulate the required conditions to fit his own objectives, the Israeli Civil Service Commissioner's report from January 2010 warned that his office cannot supervise all of the (over one hundred) openings for which auctions were announced. Thus, some of the contests are biased in order to achieve a goal which is not the maximization of the likelihood that the opening is filled with the best candidate. In our study, such alternative goal is represented by the assignments of weight to the contestants' efforts.

Note that, usually, discrimination is considered as one of the characteristics of the prevailing political culture or of the government's ideology. In contrast, in our contest setting, the weights assigned to the public well being and the contestants' efforts represent the political culture while discrimination is an endogenous variable that characterizes the mechanism allocating the prize which hinges on the political culture. Put differently, discrimination does not represent the contest designer's preferences or an imposed constraint of the political-economic environment, but rather the means he deliberately and selfishly applies to enhance his interest.

On the one hand, the law allows control on the degree of discrimination among the contestants. On the other hand, however, it protects the contestants by ensuring that their participation is minimally effective; they have some chance of "meaningful" winning if a contest is held. For this reason we restrict attention to competitive contest success functions. In particular, a take-it-or-leave-it offer to the 
contestant who values the prize more highly is not allowed because it eliminates competition and "meaningful" winning. While the ability to bias by announcing the required conditions for participation in the contest is consistent with the reality of the contest environment, the ability to choose superior yet extreme mechanisms that eliminate competition and meaningful winning are inconsistent with the basic existence of competition and real economic incentives that give rise to a contest that is beneficial for one or both of the contestants. ${ }^{5}$ The two families of contest success functions on which we focus do conform to the reality of contests as well as to the legal constraint that contests are meaningful. They can also be justified either axiomatically, in the case of Tullock lottery functions, see Clark and Riis (1998), ${ }^{6}$ or on the grounds of common use in practice. In any event these families are the most widely studied types of contest success functions in the literature.

The results can also be tested empirically. In particular, one can empirically estimate the nature and extent of discrimination and the stakes of the contestants, and thus apply our results to expose the political culture inspiring these estimates. Consider the example of monopoly power assuming that in a series of contests the government decides who will be its single supplier of a certain "product". In each period the contest designer publishes the terms of the contest and any firm that satisfies the necessary requirements can apply. Various parameters of this contest's environment can change over time: the contestants' stakes may change due to changes in the law that gives the winner more or less power and the contest designer, and, in turn, the political culture may change because of a change in the ruling political party. If the contest designer has not changed, then changes in the terms of the auction imply that discrimination changes due to a change in the contestants' stakes. We could thus relate changes in the stakes to changes in discrimination or the contest terms. On the other hand, comparing different contests with similar stakes over different periods with different contest designers enables the use of the available panel data, using dummy variables to identify the different designers and time fixed effects, in order to relate changes in the terms of the contests to changes in political culture that reflect the different contest designers. Of course many other variables

\footnotetext{
${ }^{5}$ In contrast to the situation where the designer proposes a 'take it or leave it' offer to the individual with the higher prize valuation, in our case, when there is no contest, the winner and not the designer enjoys the prize. If the designer prefers the existence of a contest, he cannot choose a mechanism that precludes the direct or indirect abolition of competition by exclusion of contestants.

${ }^{6}$ We use the same type of discrimination as in Clark and Riis (1998).
} 
would be taken into account, such as the economic state, the length of time the designer is in power, etc. To sum up, such analysis can be carried using panel data for different contests over different periods of time when both the stakes and the contest designers have changed. This analysis can be useful in examining how changes in contest designers and changes in the contestants' stakes affect discrimination.

Our first two results specify, in a two-contestant setting, the optimal discrimination from the point of view of the contest designer corresponding to any given combination of political culture and asymmetry between the contestants' valuation of the prize. These results enable the derivation of the conditions that determine whether the optimal bias is in favor of the contestant with the larger or smaller prize valuation and the investigation of the effect of a change in these parameters on discrimination. They also lead to the conclusion that within our setting, where the logit contest success function does not exhibit increasing returns to scale, an all-pay auction is always preferred to a logit contest success function from the point of view of the contest designer. This conclusion provides a new politicaleconomic micro foundation to some of the most commonly used models in the contest literature.

\section{Optimal contest design}

\subsection{The setting}

In the basic one-stage contest setting, there are two risk-neutral contestants, the high and low benefit contestants, 1 and 2 . The prize valuations of the contestants are

denoted by $n_{i}$, and with no loss of generality let $n_{1} \geq n_{2}$ or $k=\frac{n_{1}}{n_{2}} \geq 1$. Being chiefly concerned with the conditions under which the contest designer chooses an optimal contest, we assume that he has full knowledge of the contestants' prize valuations. Given these valuations and the CSF, $\operatorname{Pr}_{i}\left(x_{1}, x_{2}\right)$, the function that specifies the contestants' winning probability given their efforts $x_{1}$ and $x_{2}$, the expected net payoff of contestant $i$ is:

$$
E\left(u_{i}\right)=\operatorname{Pr}_{i}\left(x_{1}, x_{2}\right) n_{i}-x_{i},(i=1,2)
$$

As in Epstein and Nitzan (2006b, 2007), let the objective function of the contest designer in our extended setting be a weighted average of the expected social welfare and lobbying efforts: 


$$
G(\cdot)=\gamma\left[E\left(u_{1}\right)+E\left(u_{2}\right)\right]+(1-\gamma)\left(x_{1}+x_{2}\right)
$$

where the parameters $\gamma$ and $(1-\gamma)$ are the weights assigned to the expected social welfare and the contestants' lobbying outlays. These weights represent the political culture; the culture reflected by the designer's genuine objectives or the culture that imposes this objective function on the designer. The designer is assumed to maximize the objective function (2) by setting the CSF, given the Nash equilibrium efforts of the contestants. His particular choice of the CSF together with the corresponding efforts of the contestants constitute the equilibrium of the extended contest.

As in Epstein and Nitzan (2006b), the designer decides whether there is a contest on the prize or not. If there is no contest, he awards the prize to the individual with the higher prize valuation and no efforts are made by the potential contestants. Note that in contrast to the situation where the designer proposes a take-it-or-leave-it offer to the individual with the higher prize valuation, in our case, when there is no contest, the winner and not the designer enjoys the prize. If the designer prefers the existence of a contest, he has to ensure a genuine competitive environment that precludes the direct or indirect abolition of competition by exclusion of contestants ${ }^{7}$, as explained in the introduction. We therefore focus on the widely studied contests with interior equilibria that are based on all-pay-auctions and on logit CSFs.

\subsection{All-pay auctions}

In our setting, certain winning means that the designer sets a CSF that leaves no residual winning uncertainty (RWU) after the revelation of the contestants' efforts. That is, the CSF for $\delta>0$ is an all-pay auction given by:

$$
p_{1}\left(x_{1}, x_{2}\right)=\left\{\begin{array}{lll}
1 & \text { if } & x_{1}>\delta x_{2} \\
0.5 & \text { if } & x_{1}=\delta x_{2} \\
0 & \text { if } & x_{1}<\delta x_{2}
\end{array}\right.
$$

and for $\delta=0, p_{1}\left(x_{1}, x_{2}\right)=1$, where the discrimination variable $\delta \geq 0$ is selected by the contest designer. By (3), a reduction in $\delta$ increases the bias in favor of the more motivated contestant 1 . Furthermore, $0 \leq \delta<1$ implies a bias in favor of contestant 1 , with an extreme bias when $\delta=0$ (contestant 1 is the certain winner - the prize is

\footnotetext{
${ }^{7}$ For example, by applying a CSF that is always unresponsive to their effort, as in Nti (2004).
} 
awarded to the individual with the higher prize valuation). When $\delta=1$ the contest is fair, there is no bias. When $\delta>1$ the bias is in favor of contestant 2 .

The contest designer maximizes his objective function (2) by setting $\delta$, given the contest success function which is of the form given by (3) and the Nash equilibrium efforts of the contestants.

\subsection{The logit lotteries}

The widely studied contest success function of the logit form for $\delta>0$ is:

$$
p_{1}\left(x_{1}, x_{2}\right)=\frac{x_{1}^{\alpha}}{x_{1}^{\alpha}+\delta x_{2}^{\alpha}} .
$$

and for $\delta=0, p_{1}\left(x_{1}, x_{2}\right)=1$, where again $\delta \geq 0$ is selected by the contest designer. In this case, $\alpha$ can be viewed as the effect of a real unit of investment on the winning probability of a contestant. We make the standard assumption that the marginal effect of effort on the winning probability is fixed or declining. That is, $0<\alpha \leq 1$. While $\alpha$ is a given parameter, the designer, again, controls the institutional bias $\delta$ when maximizing his objective function specified in (2). Our assumption that $0<\alpha \leq 1$ is warranted since, for any $k \geq 1$ and $\delta>0$ (when $\delta=0$ there is no competition), the second order conditions of the designer's problem are only satisfied when $0<\alpha \leq 1$ (see proof of proposition 2 in the Appendix). The interpretations of the values that $\delta$ can take are the same as in sub-section 2.2.

Whether a contest is held or not, crucially depends on the parameters $k, \alpha$ and $\gamma$ as pointed out in Epstein and Nitzan (2006 b). In particular, no contest is held and the prize is awarded to the contestant with the higher prize valuation, when $\gamma>0.5$. Whenever a contest is held, our concern is focused on how the exogenous parameters $k$ and $\gamma$ determine the optimal bias in favor of one of the contestants. ${ }^{8}$ In particular, who is that contestant and how is the bias affected by changes in these parameters. The answer to these questions is not transparent because a change in $k$ and $\gamma$ might have contrasting effects on the two variables that appear in the designer's objective function.

\footnotetext{
${ }^{8}$ Note that in Epstein and Nitzan (2006b) discrimination is not a control variable of the designer.
} 


\section{Optimal discrimination}

The contest designer controls the institutional bias or the extent of the desired discrimination $\delta \geq 0$. The first preliminary result specifies the optimal discrimination $\delta^{*}$ chosen by the designer under the all-pay auction; the first type of CSF on which we focus.

Proposition 1: Under the all-pay-auction, the optimal value of discrimination is equal to $\delta^{*}=k$, if $\gamma<\frac{k+1}{3 k+1}=\gamma_{1}$, and $\delta^{*}=0$, otherwise.

Under an all-pay-auction, the contest is deterministic. In turn, the behavior of the contest designer is binary: for low values of $\gamma, \delta^{*}=k$, which means that the designer is (incompletely) in favor of contestant 2. For high values of $\gamma, \delta^{*}=0$, which means that the designer is (completely) in favor of contestant 1 . This result can be explained in the following way. For low values of $\gamma$, the designer wishes to extract the maximal efforts from the contestants. Setting the optimal bias $\delta^{*}=k$ accomplishes the designer's attempt to maximize the extent of competition between the contestants. In fact, such bias eliminates the advantage of contestant 1 and creates actual equality between the competitors. While this bias completely eliminates the contestants' surplus (the utility of each contestant is zero), it maximizes their efforts and since $\gamma$ is low, it maximizes the designer's utility. For high values of $\gamma$, the designer wishes to maximize the total utilities of the contestants. He does so by setting the optimal bias $\delta^{*}=0$, which means that contestant 1 gets the prize and no efforts are made by the contestants, so the designer's maximum utility is $m_{1}$.

The second result specifies the optimal discrimination under the logit CSF.

Proposition 2: Under the logit contest success function, the optimal value of discrimination which depends on the parameters $(k, \gamma, \alpha)$, is equal to $\delta^{*}=\frac{k^{\alpha} \Omega}{2 \gamma(k-1)+\Omega}, \quad$ if $\quad \gamma<\gamma_{0} \quad$ and $\quad \delta^{*}=0, \quad$ otherwise, where $\Omega=(k+1)(\alpha+\gamma-2 \alpha \gamma)-2 \gamma k$ and $\gamma_{0}$ solves the equation $\Omega=0$. 
By Proposition 2, under the logit CSF there is no discrimination, $\delta^{*}=1$, for some intermediate political culture $\gamma_{e}$, where $\gamma_{e}$ satisfies $\delta^{*}=1 .{ }^{9}$ As expected, when $\gamma$ is smaller than $\gamma_{e}$, the equilibrium bias is in favor of contestant 2 with the lower prize valuation, $1<\delta^{*} \leq k^{\alpha}$. In such a case the bias is incomplete. Setting such a bias is warranted because the designer assigns a sufficiently large weight to the contestants' efforts and so he increases competition and, in turn, these efforts. Note that the maximal bias in favor of contestant $2, \delta^{*}=k^{\alpha}$, is obtained for $\gamma=0$. This implies that for any level of discrimination, the winning probability of contestant 1 is not smaller than 0.5 (it is equal to 0.5 in the extreme case where $\gamma=0$ ). This is because the designer's utility is more strongly affected by the performance of contestant 1: his effort and his expected utility. Hence, for any type of designer the winning probability of contestant 1 , which is affected by the bias, is not smaller than that of contestant 2 . When $\gamma$ is larger than $\gamma_{e}$, the equilibrium bias is in favor of the more motivated contestant 1. Setting such a bias is warranted because the designer assigns a sufficiently large weight to the contestants' expected utility and so he reduces competition, which, in turn, increases the expected welfare of the contestants. Notice that $\gamma_{e}<\gamma_{0}{ }^{10}$. Therefore, when $\gamma_{0}>\gamma>\gamma_{e}$, that is, the political culture assigns a sufficiently high weight to the contestants' expected utility, thus inducing an interior equilibrium, the optimal bias in favor of contestant 1 is intermediate, $0<\delta^{*}<1$. However, when $\gamma \geq \gamma_{0}>\gamma_{e}$, the bias in favor of contestant 1 is complete, $\delta^{*}=0$. To sum up,

Corollary 1: Under the logit CSF,

When $\gamma=\gamma_{e}, \delta^{*}=1$.

When $\gamma<\gamma_{e}, 1<\delta^{*} \leq k^{\alpha}$. The bias in favor of contestant 2 is maximal, $\delta^{*}=k^{\alpha}$, for $\gamma=0$.

When $\gamma>\gamma_{e}, 0 \leq \delta^{*}<1$. The bias in favor of contestant 1 is maximal, $\delta^{*}=0$, for $\gamma \geq \gamma_{0}>\gamma_{e}$.

${ }^{9}$ Specifically $\gamma_{e}=\left[2+\frac{(k-1)\left(k^{\alpha}+1\right)}{\alpha(k+1)\left(k^{\alpha}-1\right)}\right]^{-1}$. Note that since $\frac{\partial \gamma_{e}}{\partial \alpha}>0, \operatorname{Max}_{e}=\gamma_{e}(\alpha=1)=\frac{1}{3}$.

${ }^{10}$ For this result see Appendix B. 
By Proposition 1, under the all-pay-auction, the optimal bias can have only two values; $\delta^{*}=k$ or $\delta^{*}=0$. The bias in favor of the less motivated contestant 2 is always larger than or equal to this bias under the logit CSF. Notice that for a sufficiently large $\gamma$, the bias under the two types of CSFs is equal, $\delta^{*}=0$, but the complete bias in favor of contestant 1 under the all-pay-auction requires the assignment of a larger weight of the contestants' expected utility, $\gamma_{0} \leq \gamma_{1}$.

\section{Discrimination and changes in political culture}

Utilizing the first two propositions, we now proceed to examine how changes in the political culture $(\gamma)$ affect the equilibrium bias $\delta^{*}$.

Proposition 3: In an interior equilibrium of the extended contest ${ }^{11}$, if the CSF is of the logit form, $\frac{\partial \delta^{*}}{\partial \gamma}<0$. In an interior and exterior equilibrium of the extended contest, if the CSF is an all-pay-auction, then with the exception of a neighborhood of the critical political culture $\gamma=\gamma_{1}, \frac{\partial \delta^{*}}{\partial \gamma}=0$.

Let us explain the economic intuition behind this Proposition using the formal findings of the Appendix. An increase in $\gamma$ means that the designer assigns larger significance to the public well being relative to the total efforts made by the contestants. Under the logit CSF, in equilibrium, such an increase results in more discrimination in favor of contestant 1 with the higher prize valuation, $\frac{\partial \delta^{*}}{\partial \gamma}<0$. Consequently, aggregate efforts fall because of their reduced weight in the objective function of the designer.

Notice that a bias in favor of the contestant with the higher valuation of the prize is not necessarily a bias in favor of the richer or stronger contestant. In particular, if the prize is monetary and the contestants' values represent their utilities from the prize, then since the marginal utility of income is declining, the contestant

\footnotetext{
${ }^{11}$ Since $\alpha \leq 1$, when there is an interior equilibrium under the logit CSF, $\gamma<\gamma_{0}$, and so there also exists an interior equilibrium under the all-pay-auction because $\gamma<\gamma_{1}$. Since $\gamma_{0}<\gamma_{1}$, the former inequality is therefore a sufficient condition for an interior equilibrium in both cases.
} 
with the higher valuation is the poorer one. In contrast, if the prize is not monetary, and it takes, for example, the form of the designer's commitment to improve the quality of the environment of one of the contestants, then it might be the case that the contestant with the higher prize valuation is the richer one (assuming that a rich individual values environmental quality more than a poor individual). In light of these two examples, if the CSF is of the logit form, Proposition 3 has two alternative interpretations. If the higher prize valuation is due to lower income (the first example above), then the increase in $\gamma$ intensifies affirmative action $\left(\delta^{*}\right.$ is reduced). If, however, the higher prize valuation characterizes the "stronger" (wealthier, privileged) contestant (the second example above), the increase in $\gamma$ can be viewed as weakening affirmative action in our contest setting.

Under an all-pay-auction, if $\gamma$ is sufficiently small, $\gamma<\gamma_{1}$, the designer assigns more significance to the total efforts of the contestants. Again, in this case the optimal bias $\delta^{*}=k$ accomplishes the designer's attempt to maximize the extent of competition between the contestants. In fact, such bias eliminates the advantage of contestant 1 , creates actual equality between the competitors and completely eliminates their surplus. Therefore the utility of each contestants is zero and the corresponding value of the designer's objective function, whose utility depends only on the expected aggregate efforts of the contestants in the mixed-strategy equilibrium of the contest, is $G_{A}^{*}=0.5(1-\gamma)\left(n_{1}+n_{2}\right)$. Thus, in equilibrium, the utility of the designer is equal to the average prize valuation times the weight assigned to the expected efforts. In contrast to the case of the logit CSF, under an all-pay-auction the contest is deterministic, and, in turn, the behavior of the designer is binary: for low values of $\gamma, \delta^{*}=k$. For high values of $\gamma, \delta^{*}=0$. Consequently, when $\gamma$ increases, and the equilibrium strategy of the designer is interior, his optimal discrimination remains $\delta^{*}=k$. This is due to the fact that the aggregate expected utility of the contestants remains zero and the weight assigned to total efforts, which also remain unchanged, is reduced. Note that when $\gamma$ increases, the designer can change his strategy from $\delta^{*}=k$ to $\delta^{*}=0$. In such a case contestant 1 wins the prize without making any effort and the designer's utility becomes $G_{L}^{*}=m_{1}$. This utility is still lower than the reduced utility that is equal to $(1-\gamma)$ multiplied by the equilibrium aggregate efforts corresponding to $\delta^{*}=k$ because $\gamma$ is small. That is, despite the 
reduction in his utility, when $\gamma$ increases, the designer prefers this reduced utility to the still lower utility obtained for $\delta^{*}=0$.

\section{Discrimination and changes in prize valuations}

Let us examine how changes in the asymmetry in prize valuations $(k)$ affect the equilibrium bias $\delta^{*}$ and the utility of the contest designer.

\section{Proposition 4:}

1. In an interior equilibrium of the extended contest under the logit CSF, an increase in $k$ has an equivocal effect on the equilibrium discrimination $\delta^{*}$.
a. If $\gamma<\frac{\alpha^{2}}{2 \alpha^{2}+1}\left(\leq \frac{1}{3}\right)$, then $\frac{\partial \delta^{*}}{\partial k}>0$.
b. If $\frac{\alpha}{2 \alpha+1}<\gamma$, then $\frac{\partial \delta^{*}}{\partial k}<0$. In particular, $\gamma>\frac{1}{3}$ implies that $\frac{\partial \delta^{*}}{\partial k}<0 .{ }^{12}$

2. In an interior equilibrium of the extended contest under an all-pay-auction, if $\gamma<\gamma_{1}$ (see Proposition 1), then an increase in $k$ results in an increase in the bias toward the contestant with the lower prize valuation, $\frac{\partial \delta^{*}}{\partial k}=1>0$.

For low values of $\gamma, \gamma<\frac{\alpha^{2}}{2 \alpha^{2}+1}$, an increase in $k$ increases the bias in favor of contestant 2 with the lower prize valuation, $\frac{\partial \delta^{*}}{\partial k}>0$. The reason for this is the designer's desire to make the competition more aggressive because of the large weight assigned to the contestants' efforts. Notice that the increase in the bias favoring the contestant with the lower prize valuation is relative to the situation where the bias $\delta$ remains unchanged (as before the change in $k$ ). This results in larger efforts, $\frac{\partial x_{i}^{*}}{\partial \delta}>0$, reduces expected utility of the contestant with the larger prize valuation, $\frac{\partial E\left(u_{1}^{*}\right)}{\partial \delta}<0$, and increases expected utility of the contestant with the lower prize valuation,

\footnotetext{
${ }^{12}$ This is true because $\operatorname{Max} \frac{\alpha}{2 \alpha+1}=\frac{1}{3}$ and, therefore, if $\frac{1}{3}<\gamma$, then $\frac{\alpha}{2 \alpha+1}<\gamma$.
} 
$\frac{\partial E\left(u_{2}^{*}\right)}{\partial \delta}>0$. However, the aggregate expected utility of the contestants declines, $\frac{\partial\left[E\left(u_{1}^{*}\right)+E\left(u_{2}^{*}\right)\right]}{\partial \delta}<0 .{ }^{13}$ Relative to the (inferior) situation where $\delta$ remains unchanged, the designer's utility is larger because the positive effect of the increased efforts due to the increased bias in favor of the contestant with the lower prize valuation dominates the negative effect of the reduced aggregate expected utility of the contestants. Note that the last claim does not imply that the designer's utility always increases. In particular, when $n_{2}$ is reduced, and the designer responds optimally by increasing $\delta$, his utility declines, $\frac{\partial G_{L}^{*}}{\partial n_{2}}>0$; nevertheless, it is still larger relatively to his utility in the situation where $n_{2}$ declines, yet $\delta$ remains unchanged.

For high values of $\gamma, \frac{\alpha}{2 \alpha+1}<\gamma$, an increase in $k$, the asymmetry between the prize valuations, increases the bias in favor of contestant 1 with the higher prize valuation, $\frac{\partial \delta^{*}}{\partial k}<0$. The reason for this is the designer's attempt to make the competition less aggressive because of the large weight assigned to the contestants' expected utility.

As explained in the discussion following Corollary 1, under an all-payauction, the nature of the contest is binary and therefore when $k$ increases and the equilibrium is interior, $\gamma<\gamma_{1}$, the designer becomes more biased in favor of the contestant with the lower prize valuation, $\frac{\partial \delta^{*}}{\partial k}=1>0$, in order to equalize the contestants' chances of winning, and thus induce them to increase their efforts relatively to the case where the bias remains unchanged (equal to its value before the change in $k$ ). This change in discrimination results in increased efforts of the contestants, $\frac{\partial x_{i}^{*}}{\partial \delta}>0$, in reduced expected utility of contestant $1, \frac{\partial E\left(u_{1}^{*}\right)}{\partial \delta}<0$ and no change in the expected utility of contestant $2, \frac{\partial E\left(u_{2}^{*}\right)}{\partial \delta}=0$, which means that the aggregate expected utility of the contestants declines, $\frac{\partial\left[E\left(u_{1}^{*}\right)+E\left(u_{2}^{*}\right)\right]}{\partial \delta}<0$. Relative

\footnotetext{
${ }^{13}$ For these results see Appendix B.
} 
to the situation where $\delta$ is not changed, the net effect on the designer's utility is positive because the increase in utility due to the increased efforts of the contestants dominates the reduced expected utility of the contestant with the higher prize valuation. The increased bias in favor of contestant 2 increases the designer's utility relative to the situation where he leaves the bias intact in response to the increase in $k$. Again, note that this claim does not imply that the designer's utility necessarily increases. In particular, when $n_{2}$ declines and the designer responds optimally by

increasing $\delta$, his utility declines, $\frac{\partial G_{A}^{*}}{\partial n_{2}}=0.5(1-\gamma)>0$; nevertheless, it is still larger relative to his utility in the situation where $n_{2}$ declines, yet $\delta$ remains unchanged. Also note that a change in a contestant's prize valuation positively affects the utility of the contest designer, provided that the political culture (the value of the parameter $\gamma$ ) is associated with an interior equilibrium.

Proposition 4': In an interior equilibrium, an increase in $n_{i}, i=(1,2)$ increases the utility of the contest designer.

\section{The superiority of the all-pay-auction}

In the literature, the CSF is usually assumed to be of the logit or all-pay-auction type. By Propositions 1 and 2, we get that an all-pay-auction is always the superior CSF.

Proposition 5: For any $\gamma$, the contest designer's utility under the all-pay-auction is larger than or equal to his utility under the logit CSF.

This final result provides a new political-economic micro foundation to some of the most commonly used models in the contest literature. It supports the common assumption that the CSF is an all-pay-auction because this CSF emerges as an equilibrium strategy in the extended game where the contest designer chooses between these two types of CSFs as well as determines the nature of the contest, that is, the bias in favor of one of the contestants. The contrast between Proposition 5 and Proposition 2 in Fang (2002) is due to his different model where no discrimination (exogenous or endogenous) is allowed and $\alpha=1$. The contrast between Proposition 5 and Proposition 3 in Che and Gale (1997), where the lottery contest may generate 
higher expected effort if the asymmetry is large, is caused by several differences in their modeling. Although they do not allow (exogenous or endogenous) discrimination or asymmetry in the contestants' prize valuations, they assume that the contestants have budget constraints. This implies that there is asymmetry between the differences between a contestant's prize valuation and the wealth of the second to the richest contestant which is critical in determining equilibrium under the all-payauction.

\section{Conclusion}

This study contributes new insights to the political economy of discrimination in contests. It demonstrates that political culture and asymmetry in the contested prize valuations are useful explanatory factors for understanding the diversity in the extent of discrimination in different societies. As we have seen, in the real complex contest environments, discrimination is often controlled by contest designers. The objective of our contest designer reflects his political culture and one could empirically test for our hypotheses on the nature of discrimination in contests by considering different types of contests carried out over different periods of time by different political parties or different government officials in power. By doing so one could expose, in particular, the political culture that inspires the existing bias in the existing contests (whether the discrimination is in favor of the contestant with the low or high prize valuation).

Focusing on the two most widely studied types of contest success functions (CSFs), deterministic all-pay-auctions and logit CSFs, we specify in Propositions 1 and 2 the relationship between discrimination in contests and the prevailing political culture (the weights assigned to the expected aggregate utility of the contestants and to their total efforts) as well as the asymmetry in the contestants' prize valuations. Under the logit CSF, we then derive in Corollary 1 the conditions that determine whether the optimal bias is in favor of the contestant with the larger or smaller prize valuation. It turns out that bias in favor of the more motivated contestant is driven by the assignment of sufficiently large weight to the expected utility of the contestants. In such a case, the contest designer wishes to increase the winning probability of the contestant with the larger prize valuation. Such an increase is sufficient to positively affect the total expected utility of the contestants. Bias in favor of the contestant with the lower motivation is due to the assignment of sufficiently large weight to the 
contestants' efforts. In such a case, the contest designer wishes to equalize the "strength" of the contestants and increase the extent of competition in order to induce the contestants to make larger efforts. We proceed by establishing in Proposition 3 that under a logit CSF, the bias in favor of the contestant with the higher prize valuation is increasing in the weight assigned to the expected utility of the contestants. Under an all-pay-auction, since the equilibrium bias can take only two values ( $k$ or 0$)$, the bias in favor of the more motivated contestant is almost always invariant to a change in the weight assigned to the contestants' expected utility. The effect of valuation asymmetry on the optimal bias is ambiguous. By Proposition 4, the bias in favor of the more motivated contestant is decreasing (non-decreasing) in valuation asymmetry provided that the weight assigned to the expected utility of the contestants is sufficiently small (large). By the last result, Proposition 5, an all-pay auction is always preferred to a logit CSF from the point of view of the contest designer, provided that the logit CSF is of decreasing or constant returns to scale. This finding provides a new political-economic micro rationalization to some of the most commonly used models in the contest literature. 


\section{References}

Amegashie, J.A., 2000. Some results on rent-seeking contests with shortlisting. Public Choice. 105 (3-4), 245-253.

Baik, H. B., 2008. Contests with group-specific public-good prizes. Social Choice and Welfare. 30(1), 103-17.

Baik H.B. and Lee S., 2001. Strategic groups and rent dissipation. Economic Inquiry. 39(4) 672-84.

Baye, M.R., Kovenock, D., de Vries, C., 1993. Rigging the lobbying process: an application of the all-pay auction. American Economic Review. 83, 289- 294.

Bloch F, Sánchez-Pagés S, Soubeyran R. 2006. When does universal peace prevail? secession and group formation in conflict. Economics of Governance. 7(1), 329.

Che, Y.K, Gale, I., 1997. Rent dissipation when rent seekers are budget constrained. Public Choice. 92, 109-126,

Che, Y.K, Gale, I., 1998. Caps on political lobbying. American Economic Review. 88, 643-651.

Clark, D.J., Riis, C., 1998. Contest success functions: an extension. Economic Theory. $11,201-204$.

Congleton, R.D., Hillman A.L., Konrad K.A., 2008. editors, The Theory of Rent Seeking: Forty Years of Research, Springer, Heidelberg and Berlin.

Dasgupta A. , Nti O. K., 1998. Designing an optimal contest. European Journal of Political Economy. 14( 4), 587-603.

Epstein G.S., Nitzan S., 2002. Endogenous public policy, politicization and welfare. Journal of Public Economic Theory. 4 (4), 661-677.

Epstein, G.S., Nitzan, S., 2006a. Effort and performance in public-policy contests. Journal of Public Economic Theory. 8(2), 265-282.

Epstein, G.S.,Nitzan, S., 2006b. The politics of randomness. Social Choice and Welfare. 27(2), 423-433.

Epstein, G.S., Nitzan, S., 2007. Endogenous Public Policy and Contests. Springer.

Fang, H., 2002. Lottery versus all-pay auction models of lobbying. Public Choice. 112, 351-371.

Glazer, A., Hassin, R., 1988. Optimal contests. Economic Inquiry. 26(1), 133 - 143.

Gradstein, M., 1998. Optimal contest design: volume and timing of rent seeking in contests. European Journal of Political Economy. 14(4), 575-585. 
Gradstein, M., Konrad, K., 1999. orchestrating rent seeking contests. Economic Journal. 109, 536-545.

Kahana, N., Nitzan, S., 2002. Pre-assigned rents and bureaucratic friction. Economics of Governance. 3(3), 241-248.

Konrad K.A., 2009. Strategy in contests, Oxford University Press.

Moldovanu B., Sela A., 2001. The optimal allocation of prizes in contests. American Economic Review. 91(3). 542-558

Morgan, J. Orzen H., Sefton M., 2008. Endogenous entry in contests. CeDEx Discussion Paper No. 2008-08.

Nitzan S., Ueda K., 2009. Collective contests for commons and club goods. Journal of Public Economics. 93, 48-55

Nti, K.O.. 1997. Comparative statics of contests and rent-seeking games. International Economic Review. 38(1), 43-59.

Nti, K.O., 2004. Maximum efforts in contests with asymmetric valuations. European Journal of Political Economy. 20(4), 1059-1066.

Runkel, M., 2006. Optimal contest design, closeness and the contest success function. Public Choice. 129, 217-231.

Sánchez-Pagés, S., 2007. Endogenous coalition formation in contests. Review of Economic Design. 11 (2), 139-163.

Singh, N., Wittman, D., 1998. Contest design and the objective of the contest designer: sales, promotion, sports events and patent races. in Baye's (ed.) Advances in Microeconomics. 7, JAI Press, 139-167.

Taylor, C.R., 1995. Digging for golden carrots: an analysis of research tournaments. American Economic Review. 85 (4), 872-90.

Ueda, K., 2002. Oligopolization in collective rent-seeking. Social Choice and Welfare. 19(3), 613-626. 


\section{Appendix A}

Proof Proposition 1: When $n_{1}-\delta n_{2} \geq 0$, in a mixed-strategy equilibrium, under any strategy that has a positive probability: $E\left(x_{1}+x_{2}\right)=\frac{\delta n_{2}\left(n_{1}+n_{2}\right)}{2 n_{1}}$ and $G_{A}=m_{1}+\delta \frac{n_{2}^{2}}{2 n_{1}}[(1-3 \gamma) k+1-\gamma]$. Since the inequality $(1-3 \gamma) k+1-\gamma>0 \quad$ is equivalent to $\gamma<\frac{k+1}{3 k+1}=\gamma_{1}$, we get the following result:

1. If $\gamma<\gamma_{1}$, then the optimal discrimination is the maximal $\delta$ consistent with the constraint $n_{1}-\delta n_{2} \geq 0$, which is $\delta^{*}=n_{1} / n_{2}=k$. This is an interior equilibrium of the extended all-pay-auction.

2. If $\gamma \geq \gamma_{1}$, then the optimal discrimination is $\delta^{*}=0$, that is the prize is awarded to the contestant with the higher prize valuation without a contest.

Q.E.D

Proof of Proposition 2: In equilibrium

$$
\begin{gathered}
x_{1}^{*}=\frac{\alpha \delta n_{1} k^{\alpha}}{\left(k^{\alpha}+\delta\right)^{2}}, x_{2}^{*}=\frac{\alpha \delta n_{2} k^{\alpha}}{\left(k^{\alpha}+\delta\right)^{2}} \text { and } x_{1}^{*}+x_{2}^{*}=\frac{\alpha \delta k^{\alpha}\left(n_{1}+n_{2}\right)}{\left(k^{\alpha}+\delta\right)^{2}} \\
p_{1}^{*}=\frac{k^{\alpha}}{k^{\alpha}+\delta}, p_{2}^{*}=\frac{\delta}{k^{\alpha}+\delta}, \\
E\left(u_{1}^{*}\right)=\frac{n_{1} k^{\alpha}\left[k^{\alpha}+(1-\alpha) \delta\right]}{\left(k^{\alpha}+\delta\right)^{2}} \text { and } E\left(u_{2}^{*}\right)=\frac{\delta n_{2}\left[\delta+(1-\alpha) k^{\alpha}\right]}{\left(k^{\alpha}+\delta\right)^{2}}
\end{gathered}
$$

The second order conditions of equilibrium require that $\partial^{2} E\left(u_{i}\right) / \partial x_{i}^{2}<0, i=1,2$ and the contestants' payoffs must be non-negative, that is, $E\left(u_{i}^{*}\right) \geq 0$ which requires

$$
k^{\alpha}+(1-\alpha) \delta \geq 0 \text { and } \delta+(1-\alpha) k^{\alpha} \geq 0 .
$$

A sufficient condition for the above four conditions to be satisfied is that $\alpha \leq 1$. The designer's maximal utility is therefore equal to:

$$
G_{L}=n_{2}\left\{\frac{\gamma\left[k^{2 \alpha+1}+(1-\alpha) k^{\alpha}(k+1) \delta+\delta^{2}\right]+(1-\gamma) \alpha k^{\alpha}(k+1) \delta}{\left(k^{\alpha}+\delta\right)^{2}}\right\}
$$

Suppose that the equilibrium discrimination level set by the contest designer is interior, that is, $\delta^{*}>0$ (later on we examine the possibility of $\delta^{*}=0$ ). In such a case 
his utility is maximal provided that $\frac{\partial G_{L}}{\partial \delta}=0$. This implies that the optimal bias chosen by the designer is equal to:

$$
\delta^{*}=\frac{k^{\alpha}[-2 \gamma k+(k+1)(\alpha+\gamma-2 \alpha \gamma)]}{-2 \gamma+(k+1)(\alpha+\gamma-2 \alpha \gamma)}=\frac{k^{\alpha} \Omega}{2 \gamma(k-1)+\Omega}
$$

The second order condition for this interior equilibrium is;

$$
\frac{\partial^{2} G_{L}}{\partial \delta^{2}}=\frac{-[-2 \gamma+(k+1)(\alpha+\gamma-2 \alpha \gamma)]}{\left(k^{\alpha}+\delta\right)^{3}}<0 \text { which is satisfied if: }
$$

$$
-2 \gamma+(k+1)(\alpha+\gamma-2 \alpha \gamma)>0
$$

Hence, the existence of an interior equilibrium bias, $\delta^{*}>0$, requires that the nominator in (9) is also positive. That is,

$$
\Omega=-2 \gamma k+(k+1)(\alpha+\gamma-2 \alpha \gamma)=(k+1) \alpha-\gamma[k(1+2 \alpha)+2 \alpha-1]>0
$$

Since $k>1$, we get that $k(1+2 \alpha)+2 \alpha-1>0$, which implies that the nominator in (9) is positive if:

$$
\gamma<\left[2+\frac{k-1}{\alpha(k+1)}\right]^{-1}=\gamma_{0}
$$

This inequality requires that $\gamma<0.5$. If condition (11) is not satisfied $\left(\gamma_{0} \leq \gamma \leq 1\right)$, then $\delta^{*}=0$, which means that contestant 1 is awarded the prize without any contest. In such a case $G_{L}^{*}=m_{1}$. Since $k>1$, inequality (11) implies that the SOC is satisfied. An interior equilibrium therefore exists if and only if:

$$
\gamma<\left[2+\frac{k-1}{\alpha(k+1)}\right]^{-1}=\gamma_{0}<0.5
$$

Proof of Proposition 3: Under the logit CSF, in an interior equilibrium,

$$
\frac{\partial \delta^{*}}{\partial \gamma}=-\frac{2 \alpha k^{\alpha}(k+1)(k-1)}{[-2 \gamma+(k+1)(\alpha+\gamma-2 \alpha \gamma)]^{2}}<0
$$

To expose the reason for the above result, $\frac{\partial \delta^{*}}{\partial \gamma}<0$, let us examine the effect of $\gamma$ in an interior equilibrium on the efforts of the contestants, their winning probability and their expected utility. Substituting $\delta^{*}$ (see (9)) in (5) - (7) we get: 


$$
\begin{aligned}
& x_{i}^{*}=0.25 \alpha n_{i}\left\{1-\left[\frac{(k-1) \gamma}{\alpha(k+1)(1-2 \gamma)}\right]^{2}\right\} \\
& E\left(u_{1}^{*}\right)=0.25 n_{1}\left[1+\frac{\gamma(k-1)}{\alpha(k+1)(1-2 \gamma)}\right]\left[2-\alpha+\frac{\gamma(k-1)}{(k+1)(1-2 \gamma)}\right] \\
& E\left(u_{2}^{*}\right)=0.25 n_{2}\left[1-\frac{\gamma(k-1)}{\alpha(k+1)(1-2 \gamma)}\right]\left[2-\alpha-\frac{\gamma(k-1)}{(k+1)(1-2 \gamma)}\right] \\
& p_{1}^{*}=0.5\left[\frac{\gamma(k-1)}{\alpha(k+1)(1-2 \gamma)}+1\right] \text { and (18) } p_{2}^{*}=0.5\left[1-\frac{\gamma(k-1)}{\alpha(k+1)(1-2 \gamma)}\right] \\
& \text { Hence, } \quad \frac{\partial x_{i}^{*}}{\partial \gamma}=-\frac{\alpha \gamma n_{i}}{2(1-2 \gamma)}\left[\frac{(k-1)}{\alpha(k+1)(1-2 \gamma)}\right]^{2}<0 \\
& \frac{\partial E\left(u_{1}^{*}\right)}{\partial \gamma}=0.5 n_{1} \frac{(k-1)}{\alpha(k+1)(1-2 \gamma)^{2}}\left[1+\frac{\gamma(k-1)}{(k+1)(1-2 \gamma)}\right]>0 \\
& \frac{\partial E\left(u_{2}^{*}\right)}{\partial \gamma}=-0.5 n_{2} \frac{(k-1)}{\alpha(k+1)(1-2 \gamma)^{2}}\left[1-\frac{\gamma(k-1)}{(k+1)(1-2 \gamma)}\right]<0
\end{aligned}
$$

Let us explain why $\frac{\partial E\left(u_{2}^{*}\right)}{\partial \gamma}<0$. The inequality: $\frac{\gamma(k-1)}{(k+1)(1-2 \gamma)}<1$ is equivalent to: $\gamma<\left(2+\frac{k-1}{k+1}\right)^{-1}$. Since condition (12) for an interior equilibrium is $\gamma<\left[2+\frac{k-1}{\alpha(k+1)}\right]^{-1} \quad$ and $\quad$ for $\quad$ any $\quad \alpha \leq 1$, we get that $\gamma<\left[2+\frac{k-1}{\alpha(k+1)}\right]^{-1} \leq\left(2+\frac{k-1}{k+1}\right)^{-1}$. Therefore the inequality $\frac{\gamma(k-1)}{(k+1)(1-2 \gamma)}<1$ is always satisfied in an interior equilibrium.

$$
\frac{\partial p_{1}^{*}}{\partial \gamma}>0 ; \frac{\partial p_{2}^{*}}{\partial \gamma}<0 ; \frac{\partial\left(x_{1}^{*}+x_{2}^{*}\right)^{2}}{\partial \gamma}<0 ; \frac{\partial\left[E\left(u_{1}^{*}\right)+E\left(u_{2}^{*}\right)\right]}{\partial \gamma}>0
$$

Remark: From (13) and (14) one could erroneously deduce that when $\gamma$ converges to $0.5, G_{L}^{*}$ and $x_{i}^{*}$ converge, respectively, to infinity and minus infinity. Let us show that, in fact, in an interior equilibrium $G_{L}^{*}<0.5 n_{1}$ and $0<x_{i}^{*}<0.25 \alpha n_{i}$. The reason for this is that one has to take into account that in an interior equilibrium inequality (12) is satisfied. More specifically, since for any combination of the parameters, in an 
interior equilibrium $\gamma<\left[2+\frac{k-1}{\alpha(k+1)}\right]^{-1}$ and since, as we have already shown, $\frac{\partial G_{L}^{*}(\gamma)}{\partial \gamma}>0$. We get that in such an equilibrium, $G_{L}^{*}(\gamma)<G_{L}\left[\frac{1}{2+\frac{k-1}{\alpha(k+1)}}\right]=\left[2+\frac{k-1}{\alpha(k+1)}\right]^{-1} n_{1}$. Since $\left[2+\frac{k-1}{\alpha(k+1)}\right]^{-1}<0.5$ we get that $G_{L}^{*}(\gamma)<0.5 n_{1}$. This makes sense because in an interior equilibrium, $\gamma<\left[2+\frac{k-1}{\alpha(k+1)}\right]^{-1}<0.5$. If $\gamma$ converges to 0.5 , then the expression $\frac{k-1}{\alpha(k+1)}$ converges to zero. If $\alpha$ is given, $0<\alpha \leq 1$, the expression converges to zero provided that $k$ converges to 1 . This means that for high values of $k$ the condition is not satisfied. Hence, $\gamma$ will converge to 0.5 and there will exist an interior equilibrium only when $k$ is sufficiently small (approaching 1). One cannot ask therefore what happens to $G_{L}^{*}$ when $\gamma$ converges to 0.5 , without taking into account that in such a case the permissible combinations of $k$ and $\alpha$ are reduced. As to $x_{i}^{*}$, by inequality (12) the expression within the parenthesis \{\} in (14) is positive and, therefore, $0<x_{i}^{*}<0.25 \alpha n_{i}$. For the same reason, the winning probabilities in (17) and (18) are between 0 and 1 .

When the CSF is an all-pay-auction, using the proof of Proposition 1 we get the following result:

1. If $\gamma<\gamma_{1}$, then the optimal discrimination is $\delta^{*}=\frac{n_{1}}{n_{2}}=k$. In this case,

$$
G_{A}^{*}=0.5(1-\gamma)\left(n_{1}+n_{2}\right), \frac{\partial \delta^{*}}{\partial \gamma}=0 \text { and } \frac{\partial G_{A}^{*}}{\partial \gamma}=-0.5\left(n_{1}+n_{2}\right)<0
$$

2. If $\gamma \geq \gamma_{1}$, then the optimal discrimination is $\delta^{*}=0$. In this case, $G_{A}^{*}=m_{1}$,

$$
\frac{\partial \delta^{*}}{\partial \gamma}=0 \text { and } \frac{\partial G_{A}^{*}}{\partial \gamma}=n_{1}>0
$$

Q.E.D.

Proof of Proposition 4: Let us examine the effect of a change in $k, n_{1}$ and $n_{2}$ on $\delta^{*}$ at an interior equilibrium under the logit CSF: 


$$
\frac{\partial \delta^{*}}{\partial k}=\frac{\alpha k^{\alpha-1}\left\{\left[\alpha^{2}(1-2 \gamma)^{2}-\gamma^{2}\right](k+1)^{2}-4 k \gamma(1-3 \gamma)\right\}}{[-2 \gamma+(k+1)(\alpha+\gamma-2 \alpha \gamma)]^{2}}
$$

This yields two sufficient conditions for the determination of the sign of $\frac{\partial \delta^{*}}{\partial k}$ :

a. If $\gamma<\frac{\alpha^{2}}{2 \alpha^{2}+1}$, then $\frac{\partial \delta^{*}}{\partial k}>0$. To prove the validity of this claim, notice that $k>1$ implies that $(k+1)^{2}>4 k$. Therefore, if $\alpha^{2}(1-2 \gamma)^{2}>\gamma^{2} \Leftrightarrow \alpha(1-2 \gamma)>\gamma \Leftrightarrow$ $\frac{\alpha}{2 \alpha+1}>\gamma$, then:

$$
\frac{\partial \delta^{*}}{\partial k}>\frac{\alpha k^{\alpha-1}\left\{\left[\alpha^{2}(1-2 \gamma)^{2}-\gamma^{2}\right] 4 k-4 k \gamma(1-3 \gamma)\right\}}{[-2 \gamma+(k+1)(\alpha+\gamma-2 \alpha \gamma)]^{2}}=\frac{4 \alpha k^{\alpha}(1-2 \gamma)\left[\alpha^{2}(1-2 \gamma)-\gamma\right]}{[-2 \gamma+(k+1)(\alpha+\gamma-2 \alpha \gamma)]^{2}}
$$

The last expression is positive, provided that $\alpha^{2}(1-2 \gamma)>\gamma$ or $\gamma<\frac{\alpha^{2}}{2 \alpha^{2}+1}$. Since $\frac{\alpha^{2}}{2 \alpha^{2}+1} \leq \frac{\alpha}{2 \alpha+1}, \gamma<\frac{\alpha^{2}}{2 \alpha^{2}+1}\left(\leq \frac{1}{3}\right)$ is a sufficient condition for $\frac{\partial \delta^{*}}{\partial k}>0$.

b. If $\frac{\alpha}{2 \alpha+1}<\gamma$, then $\frac{\partial \delta^{*}}{\partial k}<0$. To prove the validity of this claim, notice that $k>1$ implies that $(k+1)^{2}>4 k$. Therefore, if $\alpha^{2}(1-2 \gamma)^{2}<\gamma^{2} \Leftrightarrow \alpha(1-2 \gamma)<\gamma \Leftrightarrow$ $\frac{\alpha}{2 \alpha+1}<\gamma$, then:

$$
\frac{\partial \delta^{*}}{\partial k}<\frac{\alpha k^{\alpha-1}\left\{\left[\alpha^{2}(1-2 \gamma)^{2}-\gamma^{2}\right] 4 k-4 k \gamma(1-3 \gamma)\right\}}{[-2 \gamma+(k+1)(\alpha+\gamma-2 \alpha \gamma)]^{2}}=\frac{4 \alpha k^{\alpha}(1-2 \gamma)\left[\alpha^{2}(1-2 \gamma)-\gamma\right]}{[-2 \gamma+(k+1)(\alpha+\gamma-2 \alpha \gamma)]^{2}}
$$

The last expression is negative when $\alpha^{2}(1-2 \gamma)<\gamma$ or $\gamma>\frac{\alpha^{2}}{2 \alpha^{2}+1}$. Since $\frac{\alpha^{2}}{2 \alpha^{2}+1} \leq \frac{\alpha}{2 \alpha+1}, \frac{\alpha}{2 \alpha+1}<\gamma$ is a sufficient condition for $\frac{\partial \delta^{*}}{\partial k}<0$. In particular, this implies that for $\frac{1}{3}<\gamma, \frac{\partial \delta^{*}}{\partial k}<0$. The reason is that $\operatorname{Max} \frac{\alpha}{2 \alpha+1}=\frac{1}{3}$ and, therefore, if $\frac{1}{3}<\gamma$, then $\frac{\alpha}{2 \alpha+1}<\gamma$, which ensures that $\frac{\partial \delta^{*}}{\partial k}<0$

Let us examine now the effect of a change in $n_{1}$ on $x_{1}$.

$$
\frac{\partial x_{1}^{*}}{\partial n_{1}}=\frac{0.25 \alpha}{\alpha^{2}(1-2 \gamma)^{2}(k+1)^{3}}\left\langle\alpha^{2}(k+1)^{3}(1-2 \gamma)^{2}-\gamma^{2}(k-1)[(k-1)(k+1)+4 k]\right\rangle
$$


Hence, $\frac{\partial x_{1}^{*}}{\partial n_{1}}>0$ if and only if $\gamma<\left\{2+\frac{1}{\alpha}\left[\left(\frac{k-1}{k+1}\right)^{2}+\frac{4 k(k-1)}{(k+1)^{3}}\right]^{0.5}\right\}^{-1}$. Let us prove that this latter inequality is satisfied when $\gamma<\frac{\alpha^{2}}{2 \alpha^{2}+1}$, which also ensures that $\frac{\partial \delta^{*}}{\partial k}>0$, as proved above. Since $\alpha \leq 1, k^{3}+3 k^{2}+3 k+1>\alpha^{2}\left(k^{3}+3 k^{2}-5 k+1\right)$, which is equivalent to the following inequalities:

$$
\begin{gathered}
(k+1)^{3}>\alpha^{2}\left[\left(k^{2}-1\right)(k-1)+4 k(k-1)\right] ;(k+1)^{3}>\alpha^{2}\left\lfloor(k-1)^{2}(k+1)+4 k(k-1)\right] \\
\frac{1}{\alpha^{4}}>\frac{1}{\alpha^{2}}\left(\frac{k-1}{k+1}\right)^{2}+\frac{4 k(k-1)}{(k+1)^{3}}
\end{gathered}
$$

Taking the square root on both sides of these inequalities, we get:

$$
\begin{gathered}
\frac{1}{\alpha^{2}}>\frac{1}{\alpha}\left\{\left(\frac{k-1}{k+1}\right)^{2}+\frac{4 k(k-1)}{(k+1)^{3}}\right\}^{0.5} ; 2+\frac{1}{\alpha^{2}}>2+\frac{1}{\alpha}\left\{\left(\frac{k-1}{k+1}\right)^{2}+\frac{4 k(k-1)}{(k+1)^{3}}\right\}^{0.5} \text { thus } \\
\frac{\alpha^{2}}{2 \alpha^{2}+1}<\left\{2+\frac{1}{\alpha}\left[\left(\frac{k-1}{k+1}\right)^{2}+\frac{4 k(k-1)}{(k+1)^{3}}\right]^{0.5}\right\}^{-1}
\end{gathered}
$$

Let us proceed with the examination of the effect of a change in $n_{2}$ on $x_{2}$. Notice that inequality (12) is equivalent to the inequality:

$$
(k+1) \alpha(1-2 \gamma)>(k-1) \gamma \Leftrightarrow \alpha^{2}(k+1)^{2}(1-2 \gamma)^{2}>(k-1)^{2} \gamma^{2}
$$

Since $4 k \gamma^{2}(k-1)>0$, we get that

$$
\frac{\partial x_{2}^{*}}{\partial n_{2}}=\frac{0.25 \alpha}{\alpha^{2}(1-2 \gamma)^{2}(k+1)^{3}}\left\{(k+1)\left[\alpha^{2}(k+1)^{2}(1-2 \gamma)^{2}-(k-1)^{2} \gamma^{2}\right]+4 k \gamma^{2}(k-1)\right\}>0
$$

Consider next the effect of a change in $n_{j}$ on $x_{i}^{*}$.

$$
\frac{\partial x_{i}^{*}}{\partial n_{j}}=-0.5 \alpha n_{i} \frac{\partial k}{\partial n_{j}}\left[\frac{\gamma}{\alpha(1-2 \gamma)}\right]^{2}\left(\frac{k-1}{k+1}\right)\left[\frac{2}{(k+1)^{2}}\right]
$$

Since $\frac{\partial k}{\partial n_{1}}=\frac{1}{n_{2}}>0$ and $\frac{\partial k}{\partial n_{2}}=-\frac{n_{1}}{n_{2}{ }^{2}}<0$, we get that

$$
\frac{\partial x_{1}^{*}}{\partial n_{2}}=\frac{\alpha(k-1)}{(k+1)}\left[\frac{\gamma k}{\alpha(k+1)(1-2 \gamma)}\right]^{2}>0 ; \frac{\partial x_{2}^{*}}{\partial n_{1}}=-\frac{\alpha(k-1)}{(k+1)}\left[\frac{\gamma}{\alpha(k+1)(1-2 \gamma)}\right]^{2}<0
$$


By these results one can see that the effect of a change in $n_{2}$ on total efforts is unequivocal, $\frac{\partial\left(x_{1}^{*}+x_{2}^{*}\right)}{\partial n_{2}}>0$, but the effect of a change in $n_{1}$ is ambiguous.

$$
\frac{\partial\left(x_{1}^{*}+x_{2}^{*}\right)}{\partial n_{1}}=\frac{0.25 \alpha\left[\alpha^{2}(k+1)^{2}(1-2 \gamma)^{2}-\gamma^{2}(k-1)(k+3)\right]}{\alpha^{2}(1-2 \gamma)^{2}(k+1)^{2}}
$$

Hence, $\quad \alpha^{2}(k+1)^{2}(1-2 \gamma)^{2}>\gamma^{2}(k-1)(k+3) \Leftrightarrow \gamma<\left\{2+\frac{[(k-1)(k+3)]^{0.5}}{\alpha(k+1)}\right\}^{-1}$ implies that $\frac{\partial\left(x_{1}^{*}+x_{2}^{*}\right)}{\partial n_{1}}>0$. Let us also show that this last inequality is satisfied when $\gamma<\frac{\alpha^{2}}{2 \alpha^{2}+1}$, which guarantees that $\frac{\partial\left(x_{1}^{*}+x_{2}^{*}\right)}{\partial n_{1}}>0$ as well as $\frac{\partial \delta^{*}}{\partial k}>0$, as shown above. Since $\alpha \leq 1, \alpha^{2}\left(k^{2}+2 k-3\right)<k^{2}+2 k+1$, which is equivalent to:

$$
\begin{gathered}
\frac{\alpha^{2}}{2 \alpha^{2}+1}<\left\{2+\frac{[(k-1)(k+3)]^{0.5}}{\alpha(k+1)}\right\}^{-1} \text {. Consider now the effect of changes in } n_{1} \text { and } n_{2} \\
\text { on } G_{L} \cdot \frac{\partial G_{L}^{*}}{\partial n_{1}}=\frac{\{(k+1)[\alpha(1-2 \gamma)+\gamma]+2 \gamma\}\{(k+1)[\alpha(1-2 \gamma)+\gamma]-2 \gamma\}}{4 \alpha(1-2 \gamma)(k+1)^{2}}>0 \\
\frac{\partial G_{L}^{*}}{\partial n_{2}}=\frac{\{(k+1)[\alpha(1-2 \gamma)+\gamma]+2 \gamma k\}\{(k+1)[\alpha(1-2 \gamma)+\gamma]-2 \gamma k\}}{4 \alpha(1-2 \gamma)(k+1)^{2}}>0
\end{gathered}
$$

Both of these two last terms are positive, because due to the second order conditions, $(k+1)[\alpha(1-2 \gamma)+\gamma]-2 \gamma>0$ and in an interior equilibrium, $(k+1)[\alpha(1-2 \gamma)+\gamma]-2 \gamma k>0$.

Q.E.D

\section{Proof of Proposition 5:}

1. If $\gamma<\left[2+\frac{k-1}{\alpha(k+1)}\right]^{-1}$, (the necessary condition for an interior equilibrium under the logit CSF), then $\gamma<\left[2+\frac{k-1}{\alpha(k+1)}\right]^{-1} \leq\left(2+\frac{k-1}{k+1}\right)^{-1}$. Recall that under an all-pay-auction, if $\gamma<\left(2+\frac{k-1}{k+1}\right)^{-1}$, then $\delta^{*}=k$ and $G_{A}^{*}=0.5 n_{2}(1-\gamma)(k+1)$. Let us prove that in this case, we always obtain that $G_{L}^{*}<G_{A}^{*}$ : 


$$
n_{2} \frac{(k+1)^{2}[\alpha(1-2 \gamma)+\gamma]^{2}-4 \gamma^{2} k}{4 \alpha(k+1)(1-2 \gamma)}<0.5 n_{2}(1-\gamma)(k+1)
$$

Or, after some simplifications, $\quad \gamma<\left[2+\frac{k-1}{\sqrt{\alpha(2-\alpha)}(k+1)}\right]^{-1}$. The expression $\left[2+\frac{k-1}{\alpha(k+1)}\right]^{-1}$ (which exceeds $\gamma$, because we consider interior equilibria under the logit CSF) increases with $\alpha$. Since, for any $\alpha, 0<\alpha \leq 1$, we get that $\sqrt{\alpha(2-\alpha)} \geq \alpha$. Hence, $\gamma<\left[2+\frac{k-1}{\alpha(k+1)}\right]^{-1} \leq\left[2+\frac{k-1}{\sqrt{\alpha(2-\alpha)}(k+1)}\right]^{-1}$ which means that $G_{L}^{*}<G_{A}^{*}$. 2. If $\left[2+\frac{k-1}{\alpha(k+1)}\right]^{-1} \leq \gamma<\left(2+\frac{k-1}{k+1}\right)^{-1}$, then under the logit CSF $\delta^{*}=0$ in which case $G_{L}^{*}=m_{1}$ and under the all-pay-auction $\delta^{*}=k$ and $G_{A}=0.5 n_{2}(1-\gamma)(k+1) . \gamma<\left(2+\frac{k-1}{k+1}\right)^{-1}$ is equivalent to $G_{L}^{*}<G_{A}^{*}$.

3. If $\gamma \geq\left(2+\frac{k-1}{k+1}\right)^{-1}$, then under the two CSFs $\delta^{*}=0$ and in both cases we get that $G_{A}^{*}=G_{L}^{*}=m_{1}$.

Q.E.D

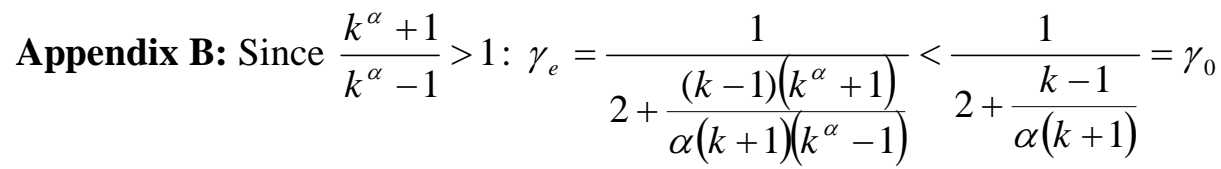

$\frac{\partial \gamma_{e}}{\partial \alpha}=\left[2+\frac{(k-1)\left(k^{\alpha}+1\right)}{\alpha(k+1)\left(k^{\alpha}-1\right)}\right]^{-2}\left(\frac{k-1}{k+1}\right) \frac{\left[2 \alpha k^{\alpha} \ln k+\left(k^{\alpha}+1\right)\left(k^{\alpha}-1\right)\right]}{\left[\alpha\left(k^{\alpha}-1\right)\right]^{2}}>0$

$\frac{\partial E\left(u_{1}\right)}{\partial \delta}=-\frac{n_{1} k^{\alpha}\left[(1+\alpha) k^{\alpha}+(1-\alpha) \delta\right]}{\left(k^{\alpha}+\delta\right)^{3}}<0, \quad \frac{\partial E\left(u_{2}\right)}{\partial \delta}=\frac{n_{2} k^{\alpha}\left[(1-\alpha) k^{\alpha}+(1+\alpha) \delta\right]}{\left(k^{\alpha}+\delta\right)^{3}}>0$

and $\frac{\partial\left[E\left(u_{1}\right)+E\left(u_{2}\right)\right]}{\partial \delta}=-\frac{n_{2}\left[\left(k^{\alpha}+\delta\right)(k-1)+\alpha\left(k^{\alpha}-\delta\right)(k+1)\right]}{\left(k^{\alpha}+\delta\right)^{3}}<0$. By (9), when $\gamma>0$

, in an interior equilibrium, $k^{\alpha}>\delta^{*}$ and therefore $\frac{\partial x_{i}}{\partial \delta}=\frac{\alpha n_{i} k^{\alpha}\left(k^{\alpha}-\delta\right)}{\left(k^{\alpha}+\delta\right)^{3}}>0$. 